\title{
On Robot Navigation Using Identical Landmarks: Integrating Measurements from a Time-of-Flight Laser
}

\author{
Ulf Larsson, Johan Forsberg and Åke Wernersson \\ Robotics \& Automation, Luleå University of Technology \\ S-971 87 LULEÅ, SWEDEN \\ Email: robotics@sm.luth.se
}

\begin{abstract}
This paper presents an algorithm for fusing scans from a time-of-flight laser and odometer readings from the robot. The range weighted Hough transform is used as a robust method to extract lines from the range data. The resulting peaks are used as feature coordinates when these lines/walls are used as landmarks during navigation. The associations between observations over the time sequence are made in a systematic way using a decision directed classifier. Natural geometrical landmarks are described in the robot frame together with a covariance matrix representing the spatial uncertainty. The map is thus built incrementally as the robot moves. If the map is given in advance the robot can find its location and navigate relative to the map.

Experimental results and simulations are presented for a mobile robot with a scanning range measuring laser with $2 \mathrm{~cm}$ resolution.
\end{abstract}

Keywords-Localization, Mobile Robot navigation, Range measuring laser, Range Weighted Hough Transform, Association, Data fusion, Telerobotics

\section{INTRODUCTION}

In this paper a systematic method is developed for estimating the position of a mobile robot relative to its environment. Current experimental tests are for indoor navigation, but the method can be extended to outdoor navigation as well.

The uncertainty in measurements and motion give rise to two different but related problems. First the model of the world and the robot position will always be uncertain. Second the identity of the observed features are unknown. The algorithm deals with both these uncertainties in a systematic way.

Previous work in this area usually use either a grid based approach (i.e. uncertainty grids [6]) or a symbolic approach where features are extracted and interpreted. Some examples of the latter can be found in [2], [12] and [13]. In the algorithm presented here the map is stored as a set of feature coordinates relative to the robot, with the corresponding covariance matrix. The estimation is performed using a Kalman filter based approach while the association uses an approximate Bayesian classifier.

The paper contains several aspects of robotics and, unfortunately, a lot of details are omitted - especially details of the approximations and several experimental tests.

\section{REPRESENTATION OF A TWO DIMENSIONAL INDOOR ENVIROMENT IN ROBOT COORDINATES}

This section introduces the model of the robots environment. The world is modelled as infinitely long straight lines (walls) described by the orthogonal distance and direction to them from the robot. The navigation algorithm is based on estimating those two parameters for each landmark. By observing those stationary landmarks at different positions the uncertainty introduced by odometric motion estimate can be reduced.

\section{A. Landmark Representation}

The map generation algorithm presented in this paper is for indoor navigation using a range measuring laser. The sensor measures the distance to objects in the horizontal plane. The most prominent features of an indoor scene are the straight lines. Those lines nearly always correspond to stationary objects such as walls.

In this paper we use the term feature to refer to the element in the scan causing the line in the range scan. The orthogonal distance $d$ and direction $\gamma$ (see fig. 1) to the line are referred as feature parameters or feature coordinates. We write the feature parameter for feature $i$ as

$$
X_{i}(k)=\left[\begin{array}{l}
d_{i}(k) \\
\gamma_{i}(k)
\end{array}\right]
$$

Assuming that the world contains some finite number $N$ of such features, then at time $t_{k}$ the landmarks in the robots environment are described by the following state vector

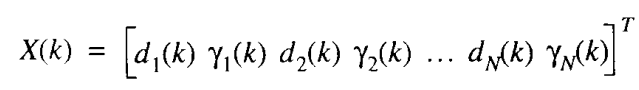

Because the landmarks are described relative to the robot it follows that there are no fixed coordinate system and no explicit representation of the robot position. The state vector could also contain other landmarks like "jump edges" at range discontinuities, corners or, for outdoor navigation, tree trunks. The algorithm below can be modified to also included these cases. 


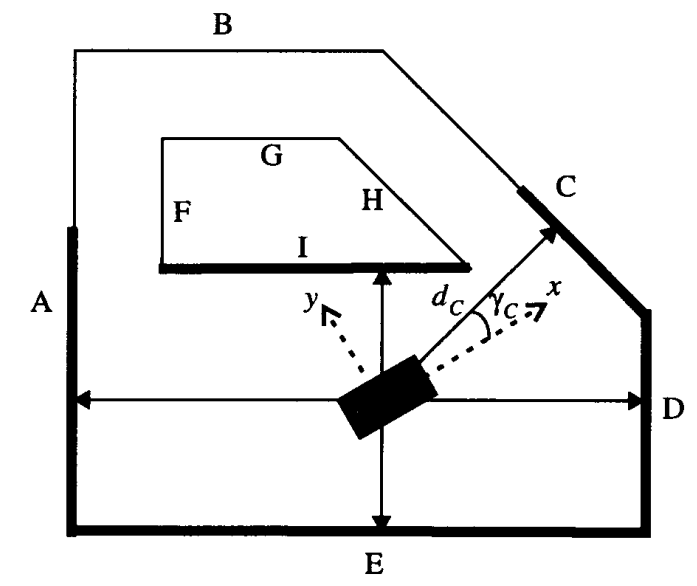

Fig. 1 Walls are described in the robot frame (the dashed arrows indicate the $\mathrm{x}$ - and $\mathrm{y}$-axes) using the orthogonal distance $d$ and the angle $\gamma$. Distances $d$ are positive when the walls are facing the robot (A,B,C,D and I), and negative when they are facing away from the robot $(\mathrm{F}, \mathrm{G}$ and $\mathrm{H})$. The feature parameters $d_{{ } \mathrm{C}}$ and $\gamma_{\mathrm{C}}$ are marked in the figure.

\section{B. The Motion Equations}

During the time period $t_{k}-t_{k-1}$ the motion is described using the displacement vector

$$
U(k)=\left[p_{x}(k) p_{y}(k) p_{\theta}(k)\right]^{T}
$$

where the two first components $\left(p_{x}, p_{y}\right)$ are the translation and the last component $p_{\theta}$ is the rotation. In the robot used during the tests, the actual motion is estimated from odometric data using the dead reckoning equations given in [10]. The state transition of the $N$ feature coordinates are

$$
X(k)=f(X(k-1), U(k))=X(k)-G(X(k-1)) U(k)
$$

where

$$
G(X(k-1))=\left[\begin{array}{ccc}
\cos \left(\gamma_{1}(k-1)\right) & \sin \left(\gamma_{1}(k-1)\right) & 0 \\
0 & 0 & 1 \\
\cos \left(\gamma_{2}(k-1)\right) & \sin \left(\gamma_{2}(k-1)\right) & 0 \\
0 & 0 & 1 \\
\ldots & \ldots & \ldots \\
\cos \left(\gamma_{N}(k-1)\right) & \sin \left(\gamma_{N}(k-1)\right) & 0 \\
0 & 0 & 1
\end{array}\right]
$$

Note that the state transition is linear in the motion $U(k)$ but not in the state vector $X(k)$. Also $U(k)$ is given in the robot frame at time $t_{k-1}$. The reader should observe that this type of formalism is possible to use also for isolated objects like tree trunks etc.

\section{Two Transformations to Fixed Coordinate Systems}

Above we described how feature are located relative to the robot. There are also cases when we want to describe a position relative to the features. To do this we introduce a local coordinate system fixed to a pair of features (lines), for example a corner in a room. This coordinate system can be used to record the robot position or to reduce the size of the estimated state vector.

Consider two non-parallel lines with parameters $\left(d_{1}, \gamma_{1}\right)$ and $\left(d_{2}, \gamma_{2}\right)$ relative to the robot. The intersection of these two lines can be used to define a origin of a ON-coordinate system. If we choose the $\mathrm{x}$-axis to be rotated $\gamma_{1}(k)+\gamma_{2}(k) / 2$ relative the robot frame then

$$
\left[\begin{array}{l}
x(k) \\
y(k) \\
\theta(k)
\end{array}\right]=\left[\begin{array}{c}
-\left(d_{1}(k)+d_{2}(k)\right) \cos \left(\frac{\gamma_{2}(k)-\gamma_{1}(k)}{2}\right) \\
\left(d_{1}(k)-d_{2}(k)\right) \sin \left(\frac{\gamma_{2}(k)-\gamma_{1}(k)}{2}\right) \\
-\frac{\gamma_{1}(k)+\gamma_{2}(k)}{2}
\end{array}\right]
$$

gives position and orientation of the robot. Other pairs of $\left(d_{i}, \gamma_{i}\right)$ in the state vector can be transformed into the new coordinate system described above.

Another coordinate system can be defined by the robots position at time $t_{o}$. Rewriting (1) and solving for the position using a least square method gives

$$
\left[\begin{array}{l}
x(k) \\
y(k) \\
\theta(k)
\end{array}\right]=\left[G(X(0))^{T} G(X(0))\right]^{-1} G(X(0))^{T}[X(0)-X(k)]
$$

\section{RANGE MEASUREMENTS AND EXTRACTED FEATURE OBSERVATIONS}

Using a time-of-flight laser on the robot a horizontal range scan is measured of its surroundings, fig. 2 . Using the range weighted Hough transform [7] the feature parameters can be found even in a cluttered room.

\section{A. The Measurement Model}

The sensor measure the distance to objects in a set of angles. The set of range measurements taken during one scan is called a range scan and denoted $\left\{r_{m} \varphi_{m}\right\}$.

A range measurement $r_{m}$ can be written as an observation of feature $i$ as

$$
r_{m}=\eta_{i}(m)\left(\frac{d_{i}}{\cos \left(\varphi_{m}-\gamma_{i}\right)}\right)+\left(1-\eta_{i}(m)\right) R\left(\varphi_{m}\right)+v_{m}
$$

where we assume that $\cos \left(\varphi_{m}-\gamma_{i}\right) \neq 0$. The binary random variable $\eta_{i}(m)$ indicates if feature $i$ is visible $\left(\eta_{i}(m)=1\right)$ or occluded $\left(\eta_{i}(m)=0\right)$. In the latter case $r_{m}$ is a measurement 


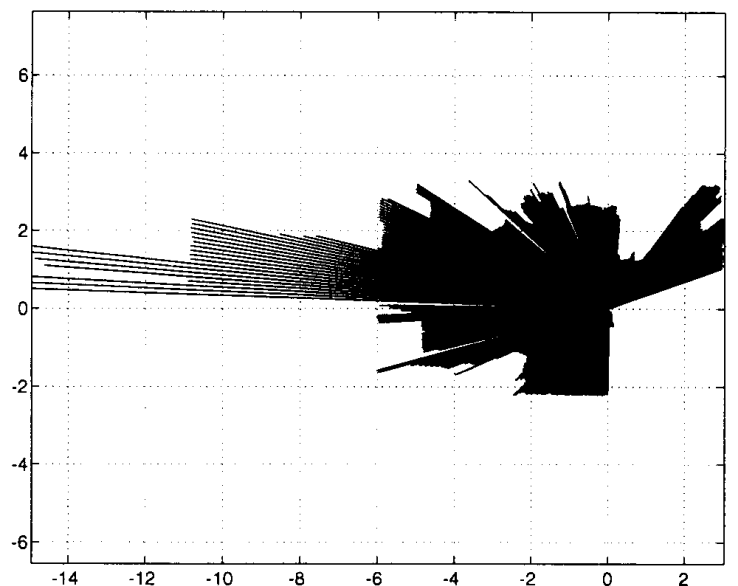

Fig. 2 Range scan of a cluttered room. Parts of the walls of the room can be seen. This is one of the rooms the robot passed through in the experiments in fig. 11

One should remember that this is not a true birds view image. The measurements are polar and taken in the plane with lots of occlusion. It is however much easier to find lines in this kind of scan than in normal images.

of the distance $R\left(\varphi_{m}\right)$ to some other object. The inaccuracy of the sensor is modelled by $v_{m}$. To estimate the feature parameters of feature $i$ we need to extract the set

$$
\left\{r_{m t} \varphi_{m} \mid\left(\eta_{i}(m)=1\right)\right\}
$$

from the range scan.

\section{B. Extracting Feature Parameters}

To extract the features parameters from the range scan we have to decide which measurements result from features and which result from disturbances. From (3) we note that in the case of $\eta_{i}(m)=1$ we get

$$
r_{m} \cos \left(\varphi_{m}-\gamma_{i}\right)-d_{i}=\nu_{m} \cos \left(\varphi_{m}-\gamma_{i}\right)
$$

Then it follows that most of the range measurements $\left(r_{m}, \varphi_{m}\right)$ where $\eta_{i}(m)=1$ satisfy the following condition

$$
\left|d_{i}-r_{m} \cos \left(\varphi_{m}-\gamma_{i}\right)\right|<h \text { where } h=3 \sqrt{\operatorname{Var}\left(v_{m}\right)}
$$

The condition given in (4) is tested for a set of $\left\{d_{i}, \gamma_{i}\right\}$ candidates. The number of candidates is chosen so that at least one pair of them are sufficiently close to the true feature parameters. If the condition is satisfied for a candidate then the measurement votes for that candidate. This is the Hough Transform. In the Range Weighted Hough Transform (RWHT) each vote is weighted with the distance $r_{m}$ to compensate for the polar range-angle data distribution. The range measurements that supported the candidate with the highest score are then used to find a least square estimate of the feature parameters. Hence we have an observation

$$
Z_{l}=\left[d_{l} \gamma_{l}\right]^{T}=X_{i}(k)+w_{l}(k)
$$

of some feature with covariance $\operatorname{Cov}\left(w_{l}\right)=S_{l}$. Approximate expressions for the covariances are given in [7]. It should be noted that we do not know the identity $i$ of the observed feature.

The process is then repeated after those measurements that satisfy the condition

$$
\left|d_{l}-r_{m} \cos \left(\varphi_{m}-\gamma_{l}\right)\right|<2 h
$$

are removed from the range scan. In this manner it is possible to find the dominating lines in the environment measured by the Laser.

In the next section we assume the following. From a range scan at time $t_{k}$ the Range Weighted Hough Transform gives us observation $Z(k)$ of $L(k)$ feature. Where

$$
Z(k)=\left[\begin{array}{lllll}
Z_{1}(k)^{T} & Z_{2}(k)^{T} & \ldots & Z_{L(k)}(k)^{T}
\end{array}\right]^{T}
$$

The corresponding covariance matrix is given by

$$
S(k)=\left[\begin{array}{cccc}
S_{1}(k) & 0_{2 \times 2} & \ldots & 0_{2 \times 2} \\
0_{2 \times 2} & S_{2}(k) & \ldots & 0_{2 \times 2} \\
\ldots & \ldots & \ldots & \ldots \\
0_{2 \times 2} & 0_{2 \times 2} & \ldots & S_{L(k)}(k)
\end{array}\right]
$$

\section{ESTIMATING THE STATE VECTOR}

The estimation of the state vector consist of two parts. First the prediction of the features coordinates based on the previous estimate and the estimate of the robots motion. Second an association function is used to associate the new observation with some feature coordinates estimated in the state vector. Then the new observation can be used to improve the estimate of the associated feature and, through the correlation, the other features. To do this the Kalman Filter formalism is used.

A critical point in our position estimation is when a measurement is to be associated to a feature in the state vector. Since there is no identity in the measurements, we need an algorithm to decide which feature the measurement belongs to. In pattern recognition this is know as unsupervised classification (see for example [5]). Because we want to build a description of the environment as the robot moves the algorithm has to be recursive.

\section{A. The State Vector}

The number of landmarks observed upto and including time $t_{k}$ is denoted $M(k)$. The state vector estimated in the Kalman filter at time $t_{k}$ consist of these $2 M(k)$ feature parameters. 


$$
\hat{X}(k)=\left[\begin{array}{c}
\hat{X}_{1}(k) \\
\hat{X}_{2}(k) \\
\ldots \\
\hat{X}_{M(k)}(k)
\end{array}\right]
$$

The estimated uncertainty of the estimate is denoted $\Sigma(k)$. The size of $\hat{X}(k)$ increases as the robot discovers new features, and might also shrink if features are discarded.

The state vector estimate can be initiated with an a priori map, or in the case of no priorinformation with the first set of peaks extracted at time $t_{0}$.

$$
\hat{X}(0)=Z(0), \Sigma(0)=S(0)
$$

Next define $H_{i}$ to be a $2 \times 2 M(k)$ matrix of the form

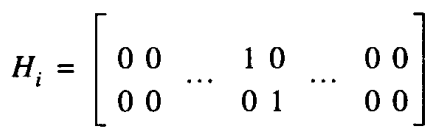

such that $\hat{X}_{i}(k)=H_{i} \hat{X}(k)$ extracts feature number $i$ from the state vector. The association problem is essentially to decide the correct matrices $H_{i}$.

\section{B. State Prediction using Odometers}

The robot motion is measured with odometers using (1) and giving the estimated displacement between $t_{k-1}$ and $t_{k}$ as

$$
\hat{U}(k)=U(k)+v(k) \quad Q(k)=\operatorname{Cov}(v(k))
$$

The prediction of the state vector is given as

$$
\begin{aligned}
& \hat{X}(k \mid k-1)=f(\hat{X}(k-1 \mid k-1), \hat{U}(k)) \\
= & \hat{X}(k-1 \mid k-1)-G(\hat{X}(k-1 \mid k-1)) \hat{U}(k)
\end{aligned}
$$

with $f()$ and $G()$ from (1).

The covariance matrix $\Sigma$ in the Extended Kalman Filter (see [1]) is then updated using the jacobian $F(k-1)$ giving the approximation

$$
\begin{aligned}
\Sigma(k \mid k-1) & \approx F(k-1) \Sigma(k-1 \mid k-1) F(k-1)^{T}+ \\
& +G(k-1) Q(k) G(k-1)^{T}
\end{aligned}
$$

Note that in the above equation $G(k-1)$ should be read as $G(\hat{X}(k-1 \mid k-1))$. To calculate $F(k-1)=\left.\frac{\partial f}{\partial X}\right|_{X=\hat{X}(k-1 \mid k-1)}$
is left to the reader. A measurement $Z_{l}$ of feature coordinates $X_{i}$ is then predicted as $H_{i} \hat{X}(k \mid k-1)$. Using the covariance matrix, we get the likelihood function for $Z_{l}$ being an observation of $X_{i}$ as

$$
P_{i}\left(Z_{l}, S_{l}\right)=\frac{e^{-\frac{1}{2}\left(Z_{l}-H_{i} \hat{X}\right)^{T}\left(H_{i} \Sigma H_{i}{ }^{T}+S_{l}\right)^{-1}\left(Z_{l}-H_{i} \hat{X}\right)}}{2 \pi \sqrt{\left|H_{i} \Sigma H_{i}{ }^{T}+S_{l}\right|}}
$$

where $S_{l}$ is the covariance of the measurement $Z_{l}$. The time index is omitted.

\section{Associating the Observations with the State Vector}

Association algorithms often uses some kind of gate around the expected value, either of a fixed size, or dependent upon the covariance for example the Mahalanobis distance. An example of using the latter method for robot navigation is given in [11]. This implies that the most probable match should always be chosen. We disagree with that sentiment and our algorithm uses the best match only if the probability is high enough, otherwise it's discarded.

From section III.B it follows that we can write observation $Z_{l}(k)$ as

$$
Z_{l}(k)=\left[\begin{array}{lllll}
\eta_{1} I & \eta_{2} I & \ldots & \eta_{N} I
\end{array}\right] X(k)+w_{l}(k)
$$

where $\eta_{1}, \eta_{2}, \ldots \eta_{N}$ are binary random variable with

$$
\sum_{i=1}^{N} \eta_{i}=1
$$

Base on previous observations we want to calculate the probability that $Z_{l}(k)$ is an observation of feature $i$.

$$
\operatorname{Pr}\left(\eta_{i}=1 \mid \hat{U}(1), \ldots, \hat{U}(k), Z(1), \ldots, Z(k-1), Z_{l}(k)\right)
$$

Using Bayes rule, a rough approximation of (7) for $1 \leq i \leq M(k)$ is given by the association function

$$
A_{i}\left(Z_{l}, S_{l}\right)=\frac{P_{i}\left(Z_{l}, S_{l}\right)}{\sum_{j=1}^{M} P_{j}\left(Z_{l}, S_{l}\right)+q}
$$

where $P_{i}\left(Z_{l}, S_{l}\right)$ is given by (6) and $q$ model the likelihood that the measurement corresponds to a feature that is not yet in the estimated state vector $(M(k)<i \leq N)$. This likelihood $q$ can be seen as the 'intensity of detectable objects' in the scene.

The function $A_{i}\left(Z_{l}, S_{l}\right)$ tell us how much we believe $Z_{l}$ is an observations of the feature $i$. In landmark based navigation it is not always desirable to use the best match as it is often very damaging to make one single mismatch but quite harmless to just skip a measurement. Thus only measurements with $A_{i}()>T$ is accepted as observations of the feature parameters $X_{i}$, where $T$ is a threshold 'close' to one. $A_{i}()$ can also be used to determine when a new measurement should be added as a new state, and when it should be discarded. An illustration of the algorithms behaviour for a simplified case is given in fig. 3 .

Above, the relations between the features coordinates were not used. In cases when the variance for each estimated feature is large but some of them are strongly correlated it might be possible to get a higher probability for the hypoth- 
esis if more than one measurement is matched simultaneously. Two examples are that we might recognize a corridor by its width or a room by its size.

For example let us look at the case of matching two features. The same approach as above can be used to associated several measurements at the same time.The corresponding association function for associating $Z_{1}$ and $Z_{2}$ with feature $i$ and $j$ respectively is given by

$$
\frac{A_{i j}\left(Z_{1}, Z_{2}\right)=}{\sum_{\substack { m_{1}=1 \\
\begin{subarray}{c}{m_{2}=1 \\
m_{2} \neq m_{1}{ m _ { 1 } = 1 \\
\begin{subarray} { c } { m _ { 2 } = 1 \\
m _ { 2 } \neq m _ { 1 } } }\end{subarray}}^{M} \sum_{m_{1} m_{2}}\left(Z_{1}, Z_{2}\right)+\sum_{m=1}^{M}\left(p_{m}\left(Z_{1}\right)+p_{m}\left(Z_{2}\right)\right) q+q^{2}}
$$

with the likelihood function for measurement of $X_{i}$ and $X_{j}$

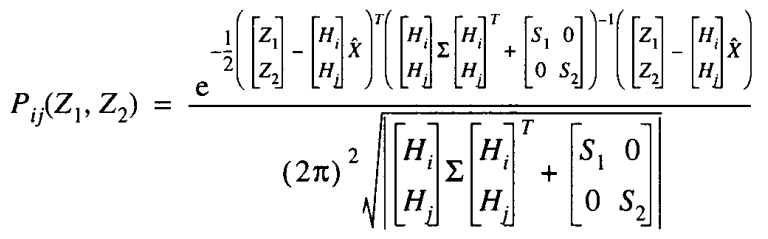

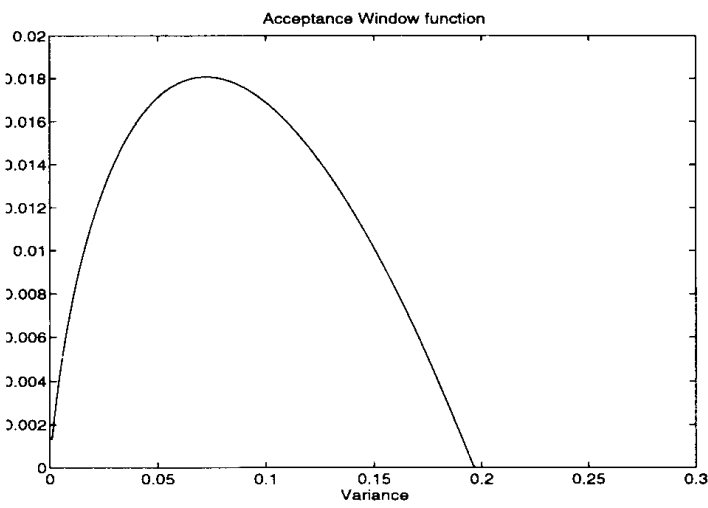

Fig. 3 This example illustrates the association algorithm for the scalar case. Let the state vector $\hat{X}$ consist of a scalar estimate $\hat{x}_{1}$ of a feature parameter $x_{1}, z$ is a possible measurement of $x_{1}$.

The corresponding one dimensional association function for this case is given by

$$
\begin{gathered}
A_{1}\left(z, \sigma^{2}\right)=\left(\frac{1}{\sqrt{2 \pi \sigma^{2}}} e^{-\frac{\left(z-\hat{x}_{1}\right)^{2}}{2 \sigma^{2}}}\right)\left(\frac{1}{\sqrt{2 \pi \sigma^{2}}} e^{-\frac{\left(z-\hat{x}_{1}\right)^{2}}{2 \sigma^{2}}}+q\right) \\
\text { with } \sigma^{2}=\operatorname{Var}\left(\hat{x}_{1}\right)+\operatorname{Var}(z)
\end{gathered}
$$

Since the new observation is independent of the prediction
$\hat{X}(k \mid k-1)$ the optimal estimate at time $k$ using information up to and including $k$ is

$$
\hat{X}(k \mid k)=\hat{X}(k \mid k-1)+K(k)\left(z_{l}(k)-H_{i} \hat{X}(k \mid k-1)\right)
$$

$$
\text { where } K(k)=H^{T}{ }_{i} \Sigma(k \mid k-1)\left(H_{i} \Sigma(k \mid k-1) H^{T}{ }_{i}+S_{l}(k)\right)^{-1}
$$

The covariance matrix is then updated by

$$
\Sigma(k \mid k)=\left(I-K(k) H_{i}\right) \Sigma(k \mid k-1)
$$

The other observations is then associated and used to update the state estimate in similar way but now $\hat{X}(k \mid k-1)$ and $\Sigma(k \mid k-1)$ is replaced with $\hat{X}(k \mid k)$ and $\Sigma(k \mid k)$ in $(6),(8),(10)$ and (11). If it not possible to associate all the measurement using a single measurement we may try to use a "higher level" of association, (9).

\section{E. Adding New Features to the State Vector}

Those observation that could not be associated to the features in the state vector might be measurement of "new" features (not estimated in $\hat{X}$ ). To avoid duplicate estimate of a single feature we require that

$$
1-\sum_{i=1}^{M} A_{i}\left(Z_{l}, S_{l}\right)>T_{N e w}
$$

where $M$ is the number of features included in $\hat{X}$. Thus the estimated probability that the observation is not from one of the estimated features has to be greater than $T_{N e w}$ for a new feature to be added.

When a new feature is added to the state vector, the state vector is augmented with the two new state variables, and the covariance matrix with the two by two covariance matrix. The covariance between the newly added states and the other states are zero. The new state vector and covariance matrix in the filter is then given by

$$
\left[\begin{array}{l}
\hat{X} \\
Z_{l}
\end{array}\right] \quad\left[\begin{array}{ll}
\Sigma & 0 \\
0 & S_{l}
\end{array}\right]
$$




\section{F. Detecting and Merging Duplicate Estimates}

The algorithms in $\mathrm{E}$. introduce new feature parameters. This might result in two separate estimates of the same feature. To detect any duplicates it is necessary to check that the estimates are well separated. This is done using the same principles as above.

To illustrate the principle assume that $\hat{X}_{M}$ and $\hat{X}_{i}$ are two estimates of the same feature parameters $X_{i} i<M$. Then we can write the estimated state vector as

$$
\hat{X}=\left[\begin{array}{c}
I \\
H_{i}
\end{array}\right]\left[\begin{array}{c}
X_{1} \\
\cdots \\
X_{M-1}
\end{array}\right]+W
$$

with $\operatorname{Cov}(W)=\Sigma$. The distance $\hat{X}_{M}-\hat{X}_{i}$ is used as a test variable in the modified association function

$$
A_{i}^{*}\left(\hat{X}_{M}\right)=\frac{P_{i}^{*}\left(\hat{X}_{M}\right)}{\sum_{j=1}^{M-1} P_{j}^{*}\left(\hat{X}_{M}\right)+q}
$$$$
\text { where } P_{i}^{*}\left(\hat{X}_{M}\right)=\frac{e^{-\frac{1}{2}\left(\hat{X}_{M}-H_{i} \hat{X}\right)}\left(\left[-H_{i} I\right] \Sigma\left[-H_{i}\right]^{T}\right)^{-1}\left(\hat{X}_{M}-H_{i} \hat{X}\right)}{2 \pi \sqrt{\left|\left[-H_{i} I\right] \Sigma\left[-H_{i} I\right]^{T}\right|}}
$$

If $A_{i}{ }^{*}\left(\hat{X}_{M}\right)>T$ then the estimated state vector is merged to $2(M-1)$ elements using

$\hat{X}^{*}=K^{*} \hat{X}$ where $K^{*}=\left(\left[I H^{T}{ }_{i}\right] \Sigma^{-1}\left[\begin{array}{c}I \\ H_{i}\end{array}\right]\right)^{-1}\left[I H^{T}{ }_{i}\right] \Sigma^{-1}$

with the covariance matrix

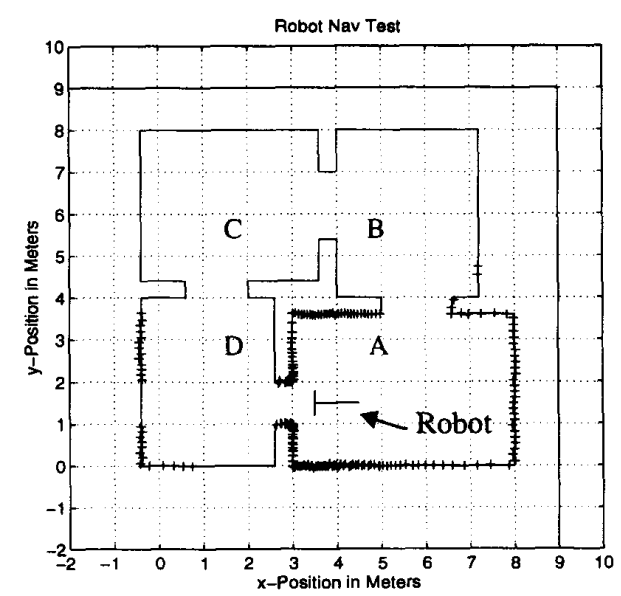

Fig. 4 In this simulation the robot moved between the four rooms, with noise added to both motion and measurements. The ' + ' signs are the random range measurements.

$$
\Sigma^{*}=K^{*} \Sigma K^{*}
$$

The merged state vector $\hat{X}^{*}$ and the covariance matrix $\Sigma^{*}$ is then used as the new estimate of the feature parameters. Note that if there are no correlation between $\hat{X}_{M}$ and the other elements in $\hat{X}$, then (12) is identical to (8) and (13)-(14) can be rewritten in the form of a Kalman Filter update (see [14]).

\section{SIMULATIONS}

For the simulations tests polygon based the environment consisted of four rooms of varying sizes, fig. 4 . The robot moved in a circle between the rooms (in the order A B C D) while the range measurements and robot kinematics were simulated. The plots illustrates typical behaviour when the motion uncertainty is large i.e. just before the algorithm breakdown.

\section{A. Navigating Using a Given A Priori Map}

The state vector was initialized with the feature coordinates for all the walls. The robot moved with constant speed and rotation. Noisy odometric readings were simulated having the following statistics for one motion step.

$$
E(\hat{U})=\left[\begin{array}{c}
0.46 \\
0.047 \\
0.20
\end{array}\right] \text { and } \operatorname{Cov}(\hat{U})=\left[\begin{array}{ccc}
0.0025 & 0.0002 & -0.0003 \\
0.0002 & 0.0023 & 0.0097 \\
-0.0003 & 0.0097 & 0.042
\end{array}\right]
$$

Using only the motion estimate to calculate the position will lead to increasing errors. The error in the estimated orientation is shown in Fig. 5.

To evaluate the navigation algorithm the estimated state vector was used to calculate the position of the robot using (2). Fig. 6 shows how the errors in the estimated orientation

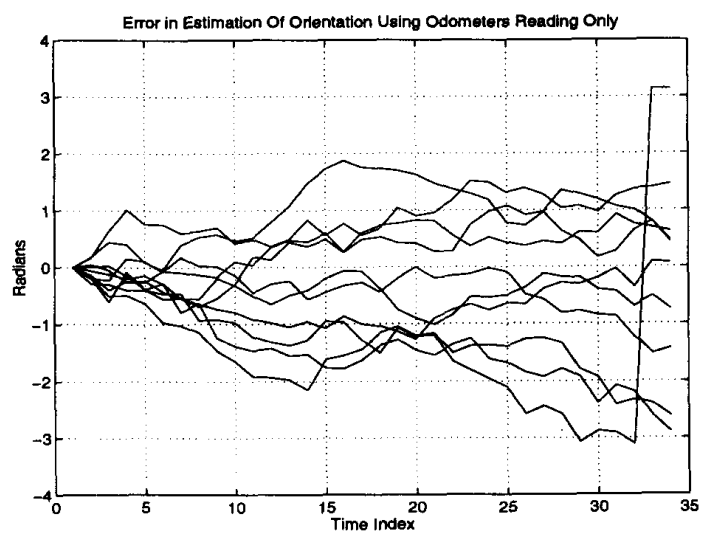

Fig. 5 The plots shows the error in the orientation of the robot when only odometric readings are used to estimate the position for 10 different simulations. At time index 33 the robot is back in the starting position and the standard deviation of the orientation error is $1.8 \mathrm{rad}$. 


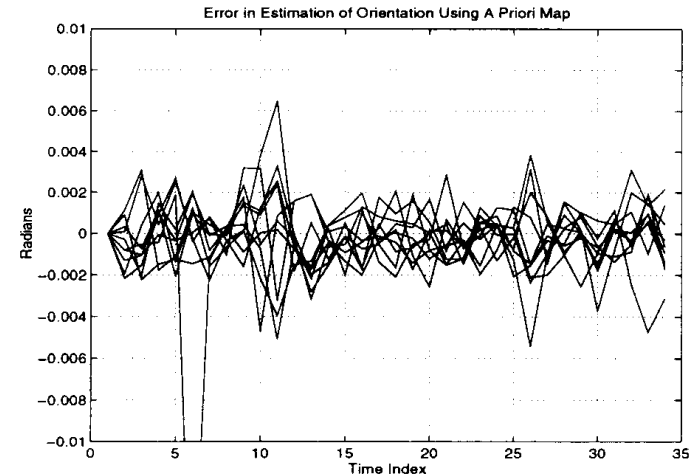

Fig. 6 This plot shows how the error in the orientation estimate is reduced when a A Priori map is used. Compare with the dead reckoning in fig. 5 .

were reduced dramatically using the algorithm compared to the earlier fig. 5 .

In fig. 7 the error in the estimated y-position is plotted. The error, as seen in the plot, increases when robot is between room $\mathrm{A}$ and $\mathrm{B}$ and between room $\mathrm{C}$ and $\mathrm{D}$. In those locations the sensor receives few measurements from the walls parallel to the $\mathrm{x}$-axis. Therefore the RWHT only extract feature coordinates corresponding to walls parallel to the y-axis. Hence there is no reduction of the "temporary dead reckoning" error in the y position.

\section{B. Navigation without an A Priori Map}

To test the map building the state vector was initialized with only two pairs of feature coordinates corresponding to the two walls forming the lower right corner in room $\mathrm{A}$. The estimates of those two were then used to calculate the robot

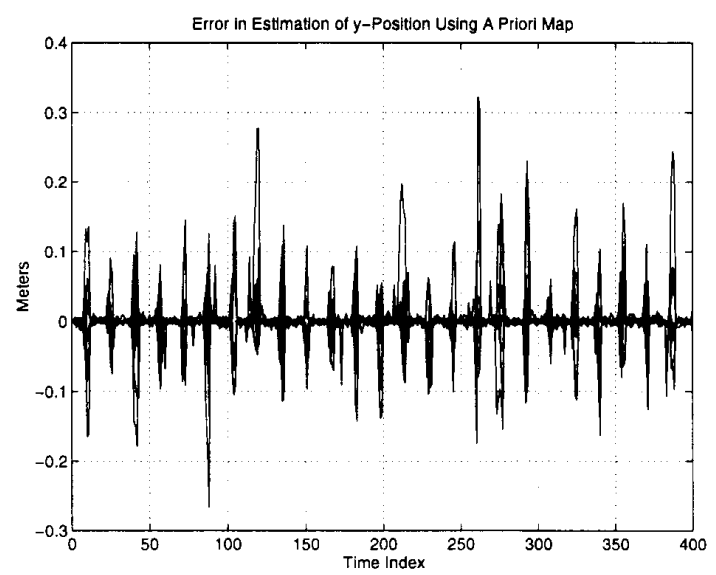

Fig. 7 Error in the $y$-position for 10 different simulations. Each run correspond to 12 1/2 lap around in the building in fig. 4.

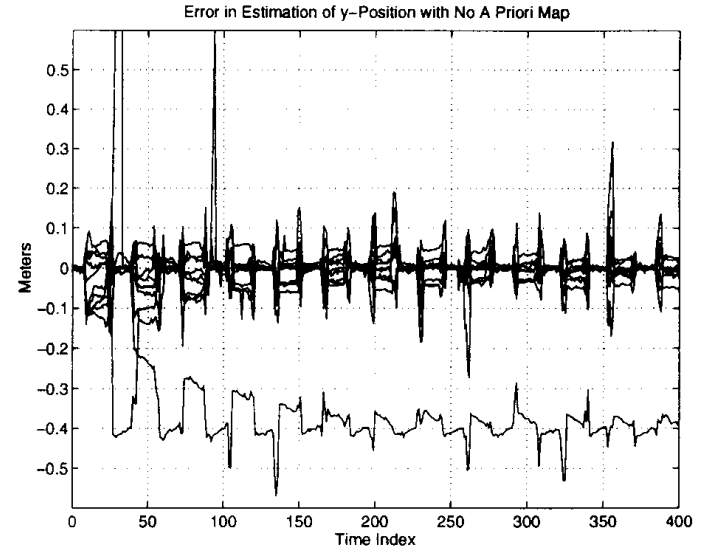

Fig. 8 Error in the $y$-position for 10 different simulations Each run correspond to 12 1/2 lap around in the building in fig. 4 A critical point when a map is created is when the robot observe features that have not been seen due to occlusion during a long time. This happens at time index 20 . In one of the test runs the algorithm failed to associate the measurements of the wall used to calculate the y-position leading to a bias in the estimate.

position. The statistics for the motion estimate were the same as in $\mathrm{A}$.

Fig. 8 shows the errors in the estimate of the y-position. If we compare with the case of an a priori map we see that the peaks in the estimation errors appears at the same position. The difference is that the error is significantly larger when the robot is in room $\mathrm{B}$ or $\mathrm{C}$ where the wall defining the $\mathrm{y}$-axis is not seen by sensor. As the robot moves the map is improved. The error in the estimated $y$-position at time index 400 is smaller than at time index 15 (the same spatial position of the robot).

The first lap when the map is created is the most critical time period. In one of the test runs shown in Fig. 8 the algorithm, after the first lap, failed to associate the measurements from the wall which was used to calculate the y-position.

\section{EXPERIMENTAL RESULTS USING A RANGE MEASURING LASER ON A MOBILE ROBOT}

The experiments were made using the mobile robot LuSAR (Luleå Semi Autonomous Robot). The tests were performed in various more or less cluttered indoor environments.

\section{A. The Mobile Robot LuSAR}

The algorithms were implemented on the mobile robot LuSAR (fig. 9) equipped with a scanning range measuring laser. The laser, an IBEO Ladar, can measure distances up to 30 meters in an 270 degree sector with resolution of 2 centimetres. Using the multitasking operating system QNX control tasks and odometric measurements can run in parallel with the time-consuming measurement processing and classification. 


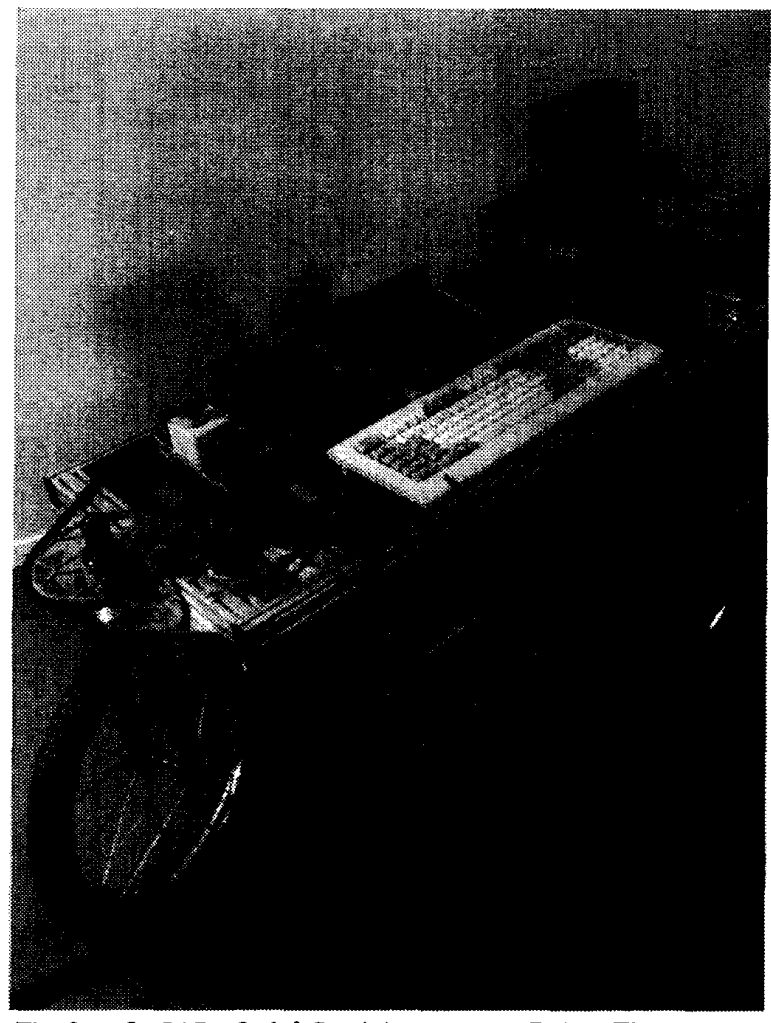

Fig. 9 LuSAR - Luleå $\underline{\text { Semi }}$ Autonomous Robot. The two rear wheels have one motor each, with a third motor for steering the single front wheel. Computations and control are performed by a standard industrial $486 \mathrm{PC}$ running a multitasking operating system (QNX). There are incremental encoders measuring the rotation of the rear wheels and the steering angle. On the top of the robot are the scanning range measuring laser. The robot is capable of speed up to $2 \mathrm{~m} / \mathrm{s}$, but most tests were performed at speed below $1 \mathrm{~m} / \mathrm{s}$ as higher speeds are impractical in the tight indoor environments.

The robot is intended to be used as a semi autonomous teleoperated robot controlled by fairly high level telecommands. A human operator selects the sequence of commands while the robot performs low-level control and planning. With increasing autonomy the bandwidth needed and the human workload will decrease. During the current localisation tests the steering of the robot was by the operator. Examples of previously tested autonomous operations are passing through doors [7] and navigating along corridors [8].The use of rate gyro was demonstrated in [9].

\section{B. Building a Map of a Laboratory with an Obstacle}

In this test a map is to be created of an empty room with a rectangular obstacle placed in the middle. The plots in fig. 10 shows how the map was generated during motion when the robot was driven around the obstacle.

To evaluate the precision the robot was driven around the lab twice, saving the state (or map) after each round. The
Table 1 Statistics from ten experimental robot runs around the laboratory in fig. 10 at 0.5 to $1 \mathrm{~m} / \mathrm{s}$. The table shows the sample standard deviation for the size of the room, and the angle between the walls. The first two column shows the results after one round around the lab, the last two the result after two rounds.

The second table shows the maximum deviations from the mean rather than the standard deviations.

\begin{tabular}{|c|c|c|c|c|}
\hline \multirow{2}{*}{$\begin{array}{c}\text { Standard } \\
\text { deviations }\end{array}$} & \multicolumn{2}{|c|}{ After one round } & \multicolumn{2}{c|}{ After two rounds } \\
Size & Angle & Size & Angle \\
\hline Long walls & $22 \mathrm{~mm}$ & $5 \mathrm{mrad}$ & $15 \mathrm{~mm}$ & $3 \mathrm{mrad}$ \\
\hline Short walls & $17 \mathrm{~mm}$ & $9 \mathrm{mrad}$ & $16 \mathrm{~mm}$ & $7 \mathrm{mrad}$ \\
\hline Diagonal wall & - & $14 \mathrm{mrad}$ & - & $9 \mathrm{mrad}$ \\
\hline
\end{tabular}

\begin{tabular}{|c|c|c|c|c|}
\hline Maximum & \multicolumn{2}{|c|}{ After one round } & \multicolumn{2}{c|}{ After two rounds } \\
\hline deviations & Size & Angle & Size & Angle \\
\hline Long walls & $37 \mathrm{~mm}$ & $7 \mathrm{mrad}$ & $24 \mathrm{~mm}$ & $6 \mathrm{mrad}$ \\
\hline Short walls & $28 \mathrm{~mm}$ & $17 \mathrm{mrad}$ & $31 \mathrm{~mm}$ & $15 \mathrm{mrad}$ \\
\hline Diagonal wall & - & $32 \mathrm{mrad}$ & - & $15 \mathrm{mrad}$ \\
\hline
\end{tabular}

speed varied between 0.5 and $1 \mathrm{~m} / \mathrm{s}$, generally higher during the second round. This process was repeated twelve times. In two of the tests the robot made some small mistakes when creating the map. Once it failed to add the short line in the upper right corner of the map, and once it added an extra wall. Neither of these faults cause any serious disturbance to the system and are in no way fatal. The ten entirely successful runs were used to calculate the statistics in table 1 .

The results conform nicely with what could be predicted. The longer walls are more precisely estimated, especially the angle, and the estimates improve after the second round about as much as can be predicted from theory. The standard deviations from the experiments are reasonably similar to those predicted by the Kalman filter.

\section{Passing through Several Cluttered Rooms}

The next test was made with the robot in two laboratories a hallway and a small room, fig. 11. The robot started in one laboratory (pos 1), moved out into a hallway (pos 2 to 4), turned back into the laboratory (pos 5) and further into the next laboratory (pos 6, 7). From there it turned back through the first laboratory and into the hallway (pos 8 ) and the back to the laboratory again (pos 9). Positions refer to the figure. The plots show range measurements taken at the nine positions overlaid onto each other using the navigation algorithm, and for comparison using only dead reckoning from the odometers.

The rooms are rather cluttered and it can be quite difficult to detect the walls in the range scan. Still the robot was capable of navigating. The robot of course used many more scans to navigate than is shown in the plots, as it continuously scans the environment while it moves. The largest disturbance was caused when the robot drove over a tube (a rotation around $x=-4$ and $y=-3$ ) 
Robot Navigation Test - Position 1

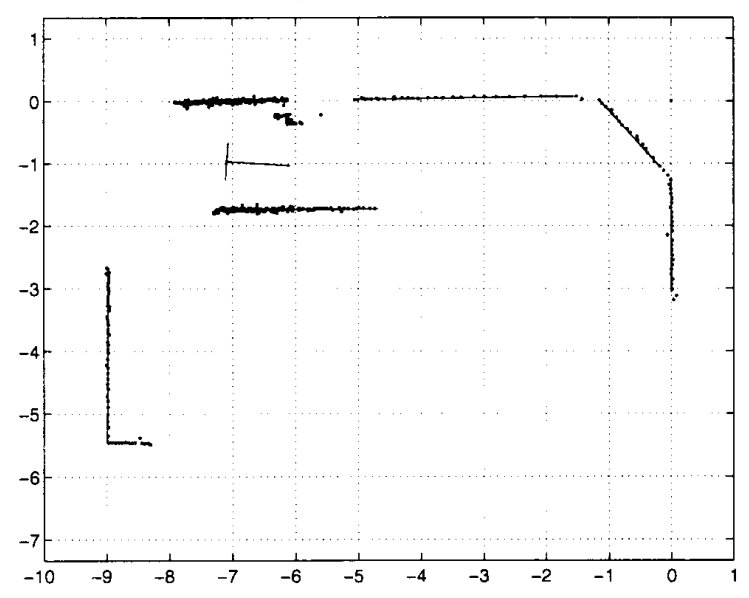

Robot Navigation Test - Position 3

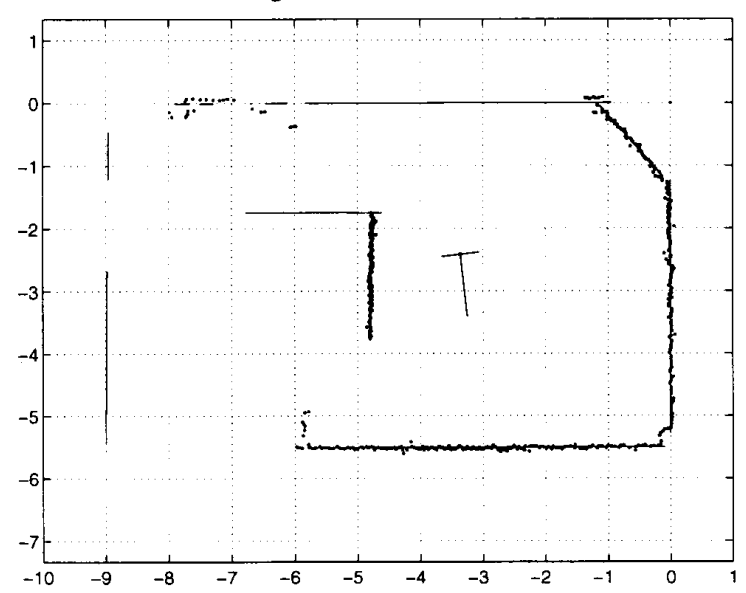

Robot Navigation Test - Position 5

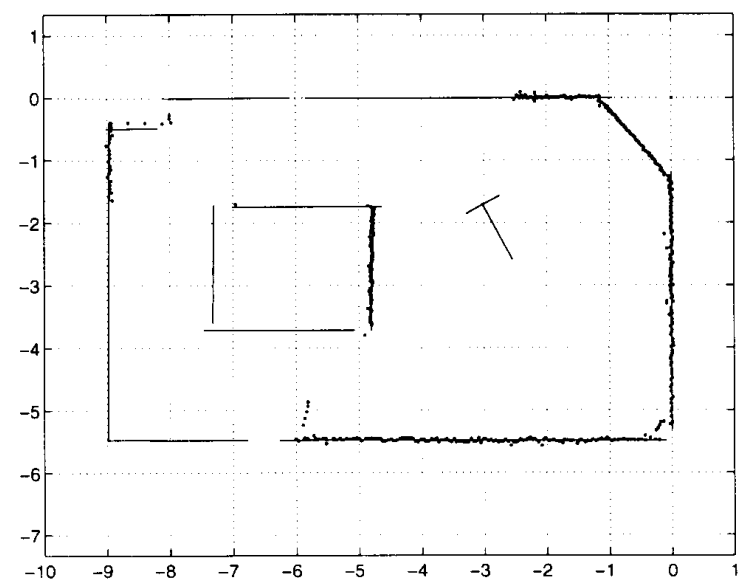

Robot Navigation Test - Position 2

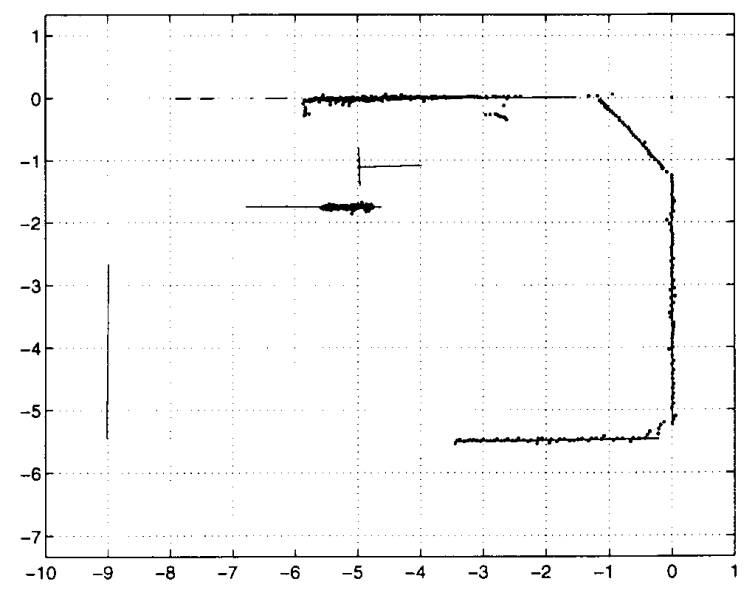

Robot Navigation Test — Position 4

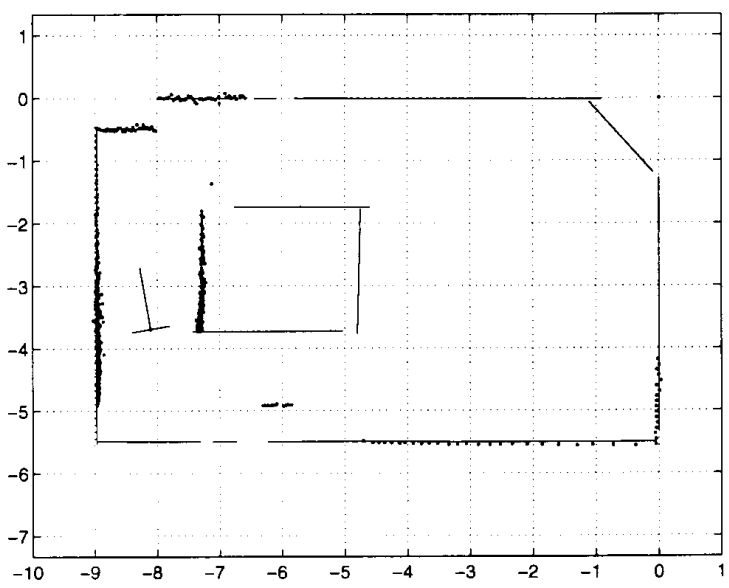

Fig. 10 Navigation test in the robot laboratory. The sequence of plots illustrate how the robot builds its map incrementally. The robot measures continuously while it moves, and made several measurements between each of the above plots.

Each of the five plots show the robot position, the current state of the map and the latest range scan. The first plot is taken before the robot started to move. Thus, all the walls in the map was generated from Hough peaks in the same range scan. The following plots show how the map is built as the robot moves around the laboratory. The final plot (5) shows the robot on its second loop around the room, successfully using the map it created on the first round. The scale is in meters.

The endpoints of the lines are currently only recorded for plotting, and not filtered. Therefore the errors in those are rather large as can be seen in the plots.

A statistical evaluation of the accuracy of the map is given in table 1. Typical standard deviations are $2 \mathrm{~cm}$.

For this test we built a rectangular obstacle in the middle of an empty room to create a test track for the robot. This test is in a very 'clean' environment to illustrate the workings of the algorithm. More cluttered tests are shown in the other examples. 


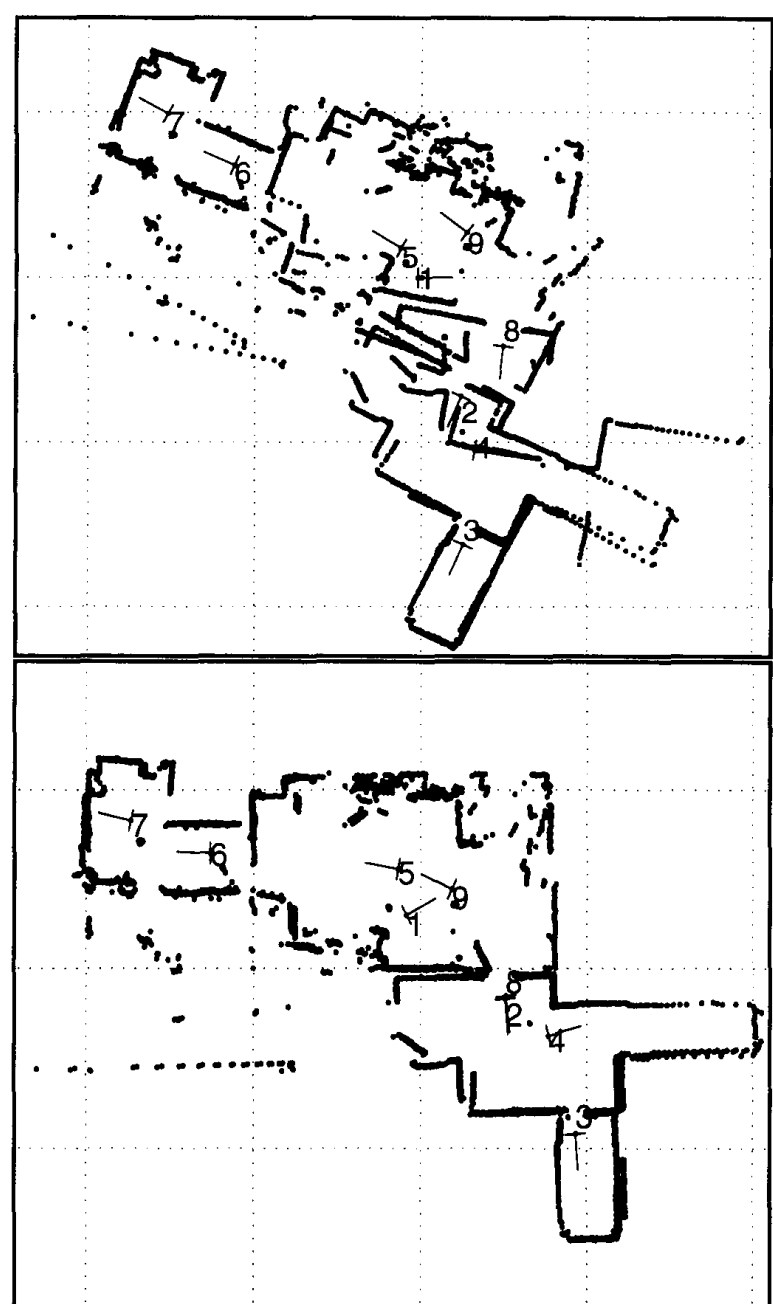

Fig. 11 The upper plot shows nine overlaid rangescans aligned using dead reckoning. The lower plot shows the same measurements but aligned using position estimates from the navigation algorithm. During the test the robot passed between the rooms as indicated by the numbered "T" symbols. The final scan taken in position 9 during this test is also shown in fig. 2 . The grid is 5 meters

\section{CONCLUSIONS AND FUTURE WORK}

The algorithm has been tested both in simulations and in several experiment. Reliable results were obtained even in cluttered environments. In a well structured environment the precision was very good, table 1 .

The reliability of the system can be exemplified by the fact that it continued to function even when, due to a mechanical fault, one rear wheel was spinning freely on its axis.

The main drawback is the computation time. For small maps, with up to 25 walls, real time operation is feasible with updating times of 1-2 seconds. As the size of the map increases the time increases quickly to unacceptable levels. A solution is to use approximations of the distributions and explore only a subset of the combinations. This will also allow simultaneous matching of more than two features, greatly increasing the robustness. Another method to improve speed is to represent states that are far away from the robot relative to other states, rather than directly relative to the robot.

Future development also include the inclusion of additional features like "jump edges" at range discontinuities, isolated objects and corners. Improved estimation of the endpoints of lines will make it possible to use the map for path planning. Matching of lines might also use the endpoints, either as a consistency test, or more closely incorporated in the probability estimation.

The navigation system will be used both in the semi autonomous robot LuSAR and in an autonomous plastering robot.

\section{REFERENCES}

[1] B.D.O. Anderson, J.B. Moore, "Optimal Filtering", Prentice Hall1979.

[2] N. Ayache, O. D. Faugeras, "Maintaining Representations of the Environment of a Mobile Robot" Autonomous Robot Vehicles. Springer Verlag, 1990.

[3] Y. Bar-Shalom, T.E. Fortmann, "Tracking and Data Association", Academic Press 1988.

[4] T. J. Broida "Kinematic and Statistical Models for Data Fusion Using Kalman Filter" Data Fusion in Robotics and Machine Intelligence. Academic Press 1992.

[5] R. O. Duda, P.E. Hart "Pattern Classification and Scene Analysis", Wiley 1973.

[6] A. Elfes, "Sonar-Based Real-World Mapping and Navigation" Autonomous Robot Vehicles. Springer Verlag, 1990.

[7] J. Forsberg U.Larsson, P. Åhman, §.. Wernersson, "The Hough Transform Inside the Feedback Loop of a Mobile Robot" IEEE Conf. Robotics and Automation, 1993, pp 791-798.

[8] J. Forsberg U.Larsson, P. Åhman, §. Wernersson, "The Hough Transform Inside the Feedback Loop of a Mobile Robot: Navigating in Corridors" SSAB Symposium on Image Processing, Uppsala February 1992

[9] T. Högström, T. Hultberg, Åke Wernersson, "A Semi Autonomous Robot with Rate Gyro Supported Control and a Video Camera" IFAC Int. Workshop Intelligent Autonomous Vehicle, Southampton 1993 pp 25-30.

[10] U. Larsson, C. Zell, K. Hyyppä, Å. Wernersson "Navigating an Articulated Vehicle and Reversing with a Trailer", IEEE Conf. Robotics and Automation, $1994 \mathrm{pp} 2398-2404$.

[11] J. Leonard, H. Durrant-Whyte, I. J. Cox, "Dynamic Map Building for an Autonomous Mobile Robot", IROS-90, pp 89-95

[12] P. Moutarlier, R. Chatila, "Stochastic Multisensory Data Fusion for Mobile Robot Location and Environment Modelling" Robotics Research 5th Int. Symp. pp 85-94

[13] R. Smith, M. Self, P. Cheeseman, "Estimating Uncertain Spatial Relationships in Robotics" Autonomous Robot Vehicles. Springer Verlag, 1990.

[14] L. Söderström, P. Stoica. "System Identification", Prentice Hall 1988. 